\title{
Protocole de suivi des populations d'aster du Saint-Laurent, Symphyotrichum laurentianum, aux Îles-de-la-Madeleine
}

\author{
GUILLAUME de LAFONTAINE
}

Département de biologie, Centre d'études nordiques, Université Laval, Québec, Québec G1K 7P4 Canada; Courriel: guillaume.de-lafontaine.1@ulaval.ca

de Lafontaine, Guillaume. 2005. Protocole de suivi des populations d'aster du Saint-Laurent, Symphyotrichum laurentianum, aux Îles-de-la-Madeleine. Canadian Field-Naturalist 119(4): 556-568.

L'aster du Saint-Laurent (Symphyotrichum laurentianum (Fernald) Nesom) est une plante endémique à la région du golfe du Saint-Laurent et on ne la retrouve qu'au Nouveau-Brunswick, à l'Île du Prince-Édouard ainsi qu'aux Îles-de-la-Madeleine, au Québec. Les populations les plus nombreuses se retrouvent aux Îles-de-la-Madeleine. L'espèce est considérée «préoccupante» par le COSEPAC depuis avril 1989 puis menacée depuis 2004. De nombreuses études réalisées par des équipes distinctes depuis 1988 ont tenté de recenser l'effectif et la superficie des populations réparties sur l'aire de distribution. Ces études étant réalisées par différentes équipes utilisant des méthodes distinctes pour mener les évaluations ont conduit à des résultats contrastants. Une revisite des occurrences connues aux Îles-de-la-Madeleine en septembre 2004 visait à mettre à jour l'état des connaissances sur la localisation exacte et l'effectif des populations d'aster sur l'archipel. Aussi, un protocole de suivi simple a été élaboré sur place, à partir duquel le personnel de l'organisme Attention Frag' ̂̂les pourra récolter les données dans le futur. Ces données pourront ensuite être cumulées et interprétées facilement par ministère développement durable environnement et parcs du Québec pour effectuer le suivi à long terme. La méthodologie, ainsi uniformisée, permettra de décrire les variations temporelles de l'état des populations en s'assurant qu'elles ne sont pas obscurcies par différentes méthodes de prise de données.

Mots clés : Aster du Saint-Laurent, Aster laurentianus, Symphyotrichum laurentianum, espèce endémique, espèce menacée, espèce rare, espèce vulnérable, golfe du Saint-Laurent, Îles-de-la-Madeleine, suivi de populations.

L'aster du Saint-Laurent est une plante endémique de la région du golfe du Saint-Laurent. On la retrouve au Nouveau-Brunswick, à l'Île du Prince-Édouard et au Québec autour des lagunes sur l'archipel des Îlesde-la-Madeleine. Puisque son aire de distribution est très limitée et qu'elle n'est répartie qu'en petites populations, l'espèce a d'abord été considérée comme «préoccupante» par le COSEPAC en avril 1989, puis son statut a été réexaminé en mai 2004 pour la désigner «menacée» (COSEPAC 2004). Ainsi, quoique décrite pour la première fois en 1914 par Fernald, les études sur la localisation et l'effectif des différentes populations n'ont véritablement débuté qu'en 1988. Depuis, différentes équipes de recherche ont étudié la situation démographique de l'aster.

Un premier rapport sur le statut de l'aster du SaintLaurent au Canada a établi les populations et leurs effectifs estimés entre 1983 et 1986 (Houle and Haber 1990). Les informations ultérieures proviennent de relevés effectués à l'Île du Prince-Édouard en 1992 et 1993 (Guignion et al. 1995) et aux Îles-de-la-Madeleine en 1994 et 1995 (Gagnon et al. 1995a, 1995b). À partir de la comparaison des résultats des études mentionnées ci-haut, Gilbert et al. (1999) soulignaient une fluctuation importante de la taille des populations dans le temps et attribuaient cette variation à la dynamique des perturbations naturelles ou anthropiques de l'habitat. Or, un troisième relevé effectué aux Îlesde-la-Madeleine entre 1999 et 2001 concluait que les populations d'aster du Saint-Laurent étaient relative- ment stables et fluctuaient peu d'une année à l'autre en absence de perturbations anthropiques (Houle et al. 2002). Enfin, suite à un relevé effectué en 2003, Duclos notait que l'aster était en déclin sur la totalité de son aire de répartition (Duclos 2004).

Ainsi, aux Îles-de-la-Madeleine, où l'on retrouve les plus grands effectifs, il y a eu quatre relevés depuis 1983. Ces quatre relevés menés par quatre équipes distinctes présentent des résultats contrastant que les auteurs interprètent différemment. Certains concluent que les populations fluctuent beaucoup (Gilbert et al. 1999; Duclos 2004), alors que d'autres, au contraire, concluent que les populations sont dans un état stable (Houle et al. 2002). On note cependant plusieurs lacunes concernant les méthodologies d'échantillonnage au sein de toutes ces études. Ainsi, ce qui constitue une population pour l'un est divisé en de nombreuses populations pour l'autre et chacun utilise sa propre numérotation pour désigner des populations définies de façon arbitraire. Aussi, les visites sur le terrain ont été effectuées à différents moments durant la saison de croissance. Sachant que l'aster est une espèce annuelle, la phénologie peut donc s'avérer d'une importance capitale pour l'estimation de la taille des populations. Pour définir l'emplacement des populations, certains utilisent des photos aériennes, une méthode rapide et pratique mais inexacte, alors que d'autres utilisent une seule coordonnée géoréférencée pour indiquer une population pouvant couvrir une superficie de plusieurs kilomètres. Dans d'autres cas, la seule information est un 
point sur une carte grossière. Par conséquent, la localisation et l'effectif des populations demeurent équivoques.

La présente étude avait pour but d'uniformiser la récolte de l'information sur l'aster du Saint-Laurent, afin de permettre au ministère du développement durable, environment et parcs du Québec de suivre à long terme les variations temporelles de l'état des populations en s'assurant qu'elles ne sont pas obscurcies par différentes méthodes de prise de données. L'organisme local Attention Frag'îles a été mandaté par le ministère du développement durable, environment et parcs afin d'assurer la collecte annuelle des données aux Îles-de-la-Madeleine.

Les objectifs spécifiques de la présente étude sont :

(1) Mettre à jour les données sur les effectifs, les localisations et les superficies des populations d'aster du Saint-Laurent aux Îles-de-la-Madeleine en 2004.

(2) Élaborer un protocole d'échantillonnage simple afin de permettre un suivi à long terme. Ce protocole a été remis au personnel d'Attention Frag'Îles basé aux Îles-de-la-Madeleine qui effectuera la récolte des données annuellement. Les données seront remises au ministère de du développement durable, environment et parcs du Québec qui assurera le suivi à long terme.

(3) Décrire la situation actuelle des populations sélectionnées pour le suivi.

\section{Espèce}

Symphyotrichum laurentianum (Fernald) Nesom est une plante halophyte annuelle, pratiquement glabre et dont la taille varie de 2 à $50 \mathrm{~cm}$ de hauteur. La tige est simple lorsque la densité est élevée ou ramifiée lorsque la densité diminue (observation personnelle). Les feuilles sont sessiles et entières, parfois un peu charnues. Les fleurs sont regroupées en capitules entourés de bractées foliacées. Un capitule est présent à chaque ramification de la tige. Les premières plantules émergent à la fin de mai et les premières inflorescences apparaissent au début du mois d'août alors que les fruits (akènes) matures sont disséminés par le vent vers la fin de septembre.

Aux Îles-de-la-Madeleine, l'espèce se retrouve typiquement sur une bande de ca. 50 à $200 \mathrm{~cm}$ de largeur en périphérie des lagunes et des marais salés (Houle et al. 2001). Cette répartition limitée est due à une dynamique particulière. Près de la rive, l'émergence de l'aster est limitée par la salinité (Houle et al. 2001, Reynolds et al. 2001; Reynolds et Houle 2002). La distribution près du rivage est également affectée par l'exposition aux vents, aux vagues lors de tempêtes, aux fortes marées d'équinoxe et aux dépôts de débris, surtout constitués de zostère (Zostera marina L.). Ce gradient de salinité et de perturbations physiques diminue en s'éloignant du rivage, favorisant ainsi la croissance de différentes espèces. Il a de plus été démontré expérimentalement que le taux de croissance relatif de l'aster du Saint-Laurent diminue lorsque la plante est placée à l'ombre (Reynolds et al. 2001; Houle et Valéry 2003). Ainsi, l'aster est rapidement remplacé par d'autres espèces plus compétitrices lorsque les conditions permettent la croissance de ces dernières (Brumbt 2001). Il s'agit donc d'une espèce tolérante à un certain niveau de stress (salinité, inondations, dépôts de zostère, vents) qui restreint les autres espèces plus compétitrices (Grime 1977, 1979).

\section{Méthodes}

Mise à jour des données sur les effectifs, les localisations et les superficies des populations

Les 25 stations de Houle et al. (2002) où l'aster était présent (entre 1999 et 2001) et une station recensée par Gagnon et al. (1995b) mais non visitée par Houle et al. ont été revisitées du 8 au 16 septembre 2004, soit pendant la période de floraison / fructification. Ce relevé inclut donc toutes les dernières populations recensées, mais exclut les occurrences historiques. Les populations ont été renommées selon des critères géographiques plutôt que numérotées arbitrairement comme dans plusieurs études précédentes. Ceci a pour but d'uniformiser la nomenclature et de classer les populations selon un critère fixe plutôt que sur une base potentiellement variable tel que la distance entre deux occurrences. L'effectif de chaque population a d'abord été estimé visuellement et une classe sur une échelle logarithmique de 0 à 6 a été attribuée à la population (classes : 0 aucun individu retrouvé; $1-1$ à 10 individus; $2-10$ à 100 individus; $3-10^{2}$ à $10^{3}$ individus; $4-10^{3}$ à $10^{4}$ individus; $5-10^{4}$ à $10^{5}$ individus; $6-10^{5}$ à $10^{6}$ individus). La localisation exacte de l'ensemble de la superficie couverte par chaque population a été définie par des coordonnées géoréférencées à l'aide d'un GPS.

\section{Élaboration d'un protocole de suivi simple}

Les trois populations ayant les effectifs les plus importants ont été sélectionnées aux fins du programme de suivi. Pour chacune des trois populations, cinq transects permanents ont été installés perpendiculairement à la ligne du rivage. Les cinq transects ont été répartis dans l'ensemble de la population de façon à couvrir l'ensemble des conditions environnementales permettant la croissance de l'aster. Tous les transects ont une dimension de $3 \mathrm{~m}$ de longueur par $1 \mathrm{~m}$ et sont divisés en six quadrats de $0.5 \mathrm{~m}$ par $1 \mathrm{~m}$. Ainsi, chaque transect couvre la largeur de la bande d'aster typiquement retrouvée le long des lagunes. Le premier quadrat se trouve près de la ligne d'eau dans le milieu plus perturbé et le dernier quadrat est situé à travers la végétation, dans le milieu plus compétitif.

Les mesures devront être prises annuellement, entre la deuxième semaine de août et la troisième semaine de septembre, soit pendant la période de floraison de l'aster. Dans chaque quadrat, huit variables importantes devront être mesurées : (1) le nombre de tiges d'aster; (2) le pourcentage de couverture par l'aster; (3) le pour- 
TABlEaU 1. Liste des occurrences d'aster du Saint-Laurent aux Îles-de-la-Madeleine en 2004 en comparaisons avec les effectifs observés dans les études précédentes. Les nombres entre parenthèses à droite du nom des populations réfèrent aux stations de Houle et al. (2002). Les chiffres en caractères gras indiquent la classe attribuée à la population dans la présente étude.

\begin{tabular}{|c|c|c|c|c|}
\hline \multirow[t]{2}{*}{ Nom de la population } & \multicolumn{4}{|c|}{ Nombre d'individus } \\
\hline & Présente étude & Duclos (2004) & Houle et al. (2002) & Gilbert et al. (1999) \\
\hline Cap de l'hôpital (11 et 12) & $4-10^{3}$ à $10^{4}$ & $10^{3}$ à $10^{4}$ & $10^{2}$ à $10^{5}$ & $10^{4}$ à $10^{5}$ \\
\hline Barachois, Chemin Poirier ( 8 et 10 ) & 1- 1 à 10 & $10^{5}$ à $10^{6}$ & 1 à 1000 & \\
\hline Barachois (9) & $5-10^{4}$ à $10^{5}$ & & $10^{5}$ à $10^{6}$ & \\
\hline Étang du nord (13) & $\mathbf{0}$ - aucun aster & Non revisitée & 1 à 1000 & $10^{5}$ à $10^{6}$ \\
\hline Havre aux Basques (14) & 5 - $10^{4}$ à $10^{5}$ & $10^{4}$ à $10^{5}$ & $+\operatorname{de} 10^{6}$ & \\
\hline Pointe des véliplanchistes $(15,16$ et 17$)$ & $3-10^{2}$ à $10^{3}$ & Non revisitée & $10^{4}$ à $10^{6}$ & \\
\hline Pointe aux canots (18) & $3-10^{2}$ à $10^{3}$ & $10^{2}$ à $10^{3}$ & $10^{5}$ à $10^{6}$ & \\
\hline Dune de l'ouest (19) & $3-10^{2}$ à $10^{3}$ & Non revisitée & $10^{6}$ à $10^{7}$ & \\
\hline Étang des caps (20) & $6-10^{5}$ à $10^{6}$ & Non revisitée & $10^{6}$ à $10^{7}$ & \\
\hline Baie du portage & 1 - 1 à 10 & Non revisitée & Non revisitée & \\
\hline Baie de Grosse Ile (43 et 44 ) & 5 - $10^{4}$ à $10^{5}$ & Non revisitée & $10^{3}$ à $10^{4}$ & Non connue \\
\hline Baie Clarke (31) & $4-10^{3}$ à $10^{4}$ & Non revisitée & $10^{3}$ à $10^{4}$ & 10 à 100 \\
\hline Pointe Old Harry (6) & $\mathbf{0}$ - aucun aster & Non revisitée & 10 à 100 & 0 à 1000 \\
\hline Bassin aux Huîtres, Bassin est (4 et 5) & $3-10^{2}$ à $10^{3}$ & 0 & $10^{3}$ à $10^{6}$ & $10^{5}$ à $10^{6}$ \\
\hline Bassin aux Huîtres, Club vacance (34) & 2 - 10 à 100 & 0 & 10 à 100 & \\
\hline Bassin aux Huîtres, centre (33) & Non revisitée & 0 & 1 à 100 & \\
\hline Bassin aux Huîtres, Bassin ouest (2 et 3 ) & $3-10^{2}$ à $10^{3}$ & $10^{3}$ à $10^{4}$ & $10^{1}$ à $10^{6}$ & \\
\hline Pointe de l'est ( 23 et 24$)$ & Non revisitée & Non revisitée & 10 à 100 & 10 à 100 \\
\hline
\end{tabular}

centage de couverture par toute la végétation; (4) le pourcentage de couverture des dépôts de zostère; (5) le pourcentage de couverture de sol nu; (6) la hauteur de la plus haute tige d'aster; (7) le nombre de capitules de la tige d'aster la plus ramifiée; (8) la richesse spécifique. Ces variables sont faciles à récolter, ne nécessitent aucun instrument de mesure autre qu'un ruban à mesurer et sont rapidement accessibles. Cependant, les mesures devront idéalement être effectuées par un observateur unique puisque les variables de recouvrement sont évaluées par estimation visuelle et sont donc sujettes à changer selon l'observateur. Les variables sélectionnées donnent un indice de l'effectif, la densité, l'habitat et la morphologie de la population à l'étude.

Description de la situation actuelle aux populations sélectionnées pour le suivi

Les mesures aux trois populations ont été prises selon la méthode décrite à l'objectif précédent entre le 16 et le 21 septembre 2004. Les analyses statistiques ont été effectuées à l'aide du logiciel Statistica release 5 (StatSoft, Tulsa, Oklahoma, USA). Il est à noter que les variables pourcentage de couverture du dépôt de zostère et pourcentage de couverture de sol nu n'ont pas été utilisées dans les analyses. Ces variables fournissent un indice de l'habitat, mais ne seront pas discutées en détail.

Une analyse de variance nichée de modèle II pur (pure Model II nested ANOVA) où le facteur transect est niché dans le facteur population a été effectuée (Sokal et Rohlf 1969). Ceci permet de déceler l'existence de véritables différences entre les populations d'une part et les cinq transects de chaque population d'autre part. Pour toutes les variables générant des résultats significatifs $(\alpha=0.05)$, une comparaison des moyennes à l'aide du test Tukey HSD a été effectuée entre les populations.

Les données des trois populations ont ensuite été analysées pour vérifier l'existence d'une relation entre chaque variable et l'éloignement par rapport à la ligne d'eau. La meilleure régression, linéaire ou quadratique, a été sélectionnée à chaque population et pour chaque variable afin d'expliquer la relation. Les critères de sélection pour la meilleure régression était la puissance du coefficient de régression, le raisonnement de l'interprétation mathématique et la signification biologique.

\section{Résultats}

Mise à jour des données sur les effectifs, les localisations et les superficies des populations

L'aster était présent à toutes les stations revisitées sauf deux. De plus, deux stations n'ont pas été revisitées dû aux contraintes de temps (Pointe de l'Est) et à l'établissement d'une résidence privée (Bassin aux huîtres - secteur centre). Le tableau 1 présente les populations et leurs effectifs en comparaison des occurrences notées dans Duclos (2004), Houle et al. (2002) et Gilbert et al. (1999). Les études de Houle et Haber (1990) et Gagnon et al. (1995a, 1995b) sont incluses dans Gilbert et al. (1999).

Premièrement, il est à noter que l'étude de Houle et al. (2002) divise les populations (25 stations) alors que Gilbert et al. (1999) tend à les regrouper (8 occurrences). Duclos (2004) a utilisé la numérotation de Houle et al. (2002). La présente étude propose une nouvelle définition des populations issues des stations de 
Houle et al. (2002). Quelques stations qui n'étaient pas clairement divisées sur le terrain ont été regroupées. Ainsi, l'étude actuelle définit 18 populations nommées selon des références géographiques plutôt que numérotées arbitrairement. Les informations relatives à chaque populations sont présentées ci-après:

\section{Cap de l'hôpital \\ Classe 4 ( \pm 1500 individus) \\ Localisation : $\mathrm{N} 47^{\circ} 25.112^{\prime}$ et $\mathrm{N} 47^{\circ} 25.100^{\prime}$ \\ W 61 $53.808^{\prime}$ W 61ํำ $53.933^{\prime}$}

Cette population a décliné depuis les premières observations de Houle et al. (2002). Un ensablement du site a entraîné une diminution considérable de l'effectif en 2001. Les observations récentes (Duclos 2004 et cette étude) montrent que la population a survécu à cette perturbation, mais que son effectif n'est pas encore retourné aux valeurs déjà observées.

\section{Barachois, Chemin Poirier \\ Classe 1 (4 individus) \\ Localisation : $\mathrm{N} 47^{\circ} 25.180^{\prime}$ \\ W $61^{\circ} 51.971^{\prime}$}

Petite population spatialement distincte de la grande population Barachois. Seulement quelques individus répartis très localement. Houle et al. (2002) ont observé une population de classe 3 en 1999 et 2000, alors qu'en 2001 la population était de classe 2 . Ce présent relevé note une population de classe 1 . Ainsi, cette population montre une tendance continuelle au déclin depuis les premières observations.

\section{Barachois \\ Classe 5 ( \pm 63000 individus) \\ Localisation : N $47^{\circ} 25.440^{\prime}$ à N $47^{\circ} 25.365^{\prime}$ \\ W $61^{\circ} 52.015^{\prime} \mathrm{W} 61^{\circ} 51.689^{\prime}$}

La population commence au bout d'un chemin (début de la grande plage) et est présente dans les premiers mètres de végétation à l'interface entre la grande plage et le barachois. La population démontre une grande variabilité phénotypique. À la pointe du barachois les individus sont plus hauts et ramifiés et sont répartis en tiges isolées. Vers le début de la grande plage, les individus sont petits, peu ramifiés et regroupés en touffes denses. L'habitat est soumis à des perturbations naturelles (inondation, ensablement) et anthropiques (VTT véhicules tout-terrain). En comparaison avec les études précédentes, l'ordre de grandeur de cette importante population apparaît relativement stable. Gilbert et al. (1999) décrivait une population de classe 5, alors que Houle et al. (2002) et Duclos (2004) l'ont classée 6. Dans la présente étude, la population est reclassée 5 . Cette population a peut-être été sousévaluée ou surévaluée en raison de la difficulté d'estimation due à la superficie et l'effectif considérables. Ainsi, en l'absence d'une tendance continue entre les études, il est difficile de poser des conclusions quant à la dynamique de cette population. Le protocole de suivi à long terme inclura ce site afin de déterminer si les différences observées proviennent de différentes méthodes d'estimation ou de tendances réelles.

\section{Étang du Nord}

Classe 0, aucun individu retrouvé à ce site (station 13 de Houle et al. (2002)). En 2001, Houle et al. (2002) ont constaté un déclin de cette population qui passait de la classe 3 à la classe 1 . Trois ans plus tard, il est possible que cette population ait décliné jusqu'à l'extinction.

\section{Havre aux Basques}

Classe 5 ( \pm 64000 individus)

Localisation : N $47^{\circ} 18.394^{\prime}$ à N47º $17.465^{\prime}$

$$
\text { W } 61^{\circ} 56.329^{\prime} \mathrm{W}^{\circ} 61^{\circ} 56.080^{\prime}
$$

La population est répartie en deux zones le long de la lagune. Dans la première zone, quelques tiges isolées sont retrouvées dans les sites favorables. Le littoral est tranché par l'érosion et les dépôts de zostère à plusieurs endroits ce qui diminue le nombre de sites favorables à l'établissement de l'aster. La seconde zone compte une plus grande densité d'individus et est caractérisée par des tiges plus ramifiées et plus hautes. Le milieu est apparu moins perturbé. Ici encore, les estimés ne sont pas constants entre les études et aucune tendance ne peut être décelée. Comme la population du Barachois, celle-ci sera inclue dans le suivi.

\section{Pointe des véliplanchistes}

Classe 3 ( \pm 200 individus)

Localisation : $\mathrm{N}^{\prime} 7^{\circ} 16.928^{\prime}$ à $\mathrm{N} 47^{\circ} 15.816^{\prime}$

$$
\text { W61 } 55.808^{\prime} \mathrm{W} 61^{\circ} 55.632^{\prime}
$$

La population peu nombreuse couvre une vaste étendue très perturbée par les inondations. Par rapport à la seule autre étude qui traite spécifiquement de cette population (Houle et al. 2002), l'effectif a diminué considérablement passant d'une population de classe 6 à une population de classe 3. Ceci indique un changement certain de l'effectif de cette population indépendant des méthodes utilisées pour l'estimation. Cependant, puisque aucune autre étude ne s'attarde spécifiquement à cette population, on ne peut déterminer s'il s'agit d'une véritable tendance au déclin.

\section{Pointe aux canots}

Classe 3 ( \pm 100 individus)

$$
\text { Localisation : } \mathrm{N} 47^{\circ} 15.959^{\prime} \text { à } \mathrm{N} 47^{\circ} 16.016^{\prime}
$$$$
\text { W61 }{ }^{\circ} 58.569^{\prime} \text { W61 } 58.694 \text { ' }
$$

La petite population est soumise à des perturbations (inondation, dépôt de zostère). Lors d'une comparaison entre les sites de Pointe aux canots et Havre aux Basques, Reynolds et Houle (2002) observaient que la population de Pointe aux canots était dans une baie protégée. Cependant, lors du relevé de 2004, j'ai pu observer que de nombreux individus étaient ensevelis sous d'épais dépôts de zostère. L'essentiel de la végétation croissait immédiatement après la ligne d'eau sur le littoral, ne laissant que peu de micro-sites favorables à l'établissement de l'aster. L'étude de Houle et al. (2002) comptait beaucoup plus d'individus que l'étude de Duclos (2004) et la présente étude. En effet, selon Houle et al. (2002), la population était de classe 6 alors que les relevés suivants présentent une population de classe 3 . Tel que mentionné pour la population précédente, ceci indique un changement certain de l'effectif indépendant des méthodes utilisées pour l'estimation. De plus, puisque la diminution est constante au cours de deux études subséquentes, il semble que la population soit en déclin.

\section{Dune de l'ouest}

Classe 3 ( \pm 100 individus)

Localisation : $N 47^{\circ} 19.429^{\prime}$ à N47 $19.377^{\prime}$

W61 ${ }^{\circ} 57.448^{\prime}$ à W61 $57.482^{\prime}$

L'étude de Houle et al. (2002) recensait plus de 2 millions d'individus dans cette population et l'occurrence polygonale notée par le centre de données sur le patrimoine naturel du Québec (CDPNQ) (Gagnon et al. 1995b) était de superficie considérable. Or, en 2004, seulement une centaine d'individus 
ont été retrouvés regroupés dans une zone très restreinte. Le milieu ne présente pas une inclinaison importante et il s'agit plutôt d'une vaste plaine hautement productive. Lors de la visite, toute cette plaine était inondée et des espèces parfois retrouvées en compétition avec l'aster et non aquatiques (e.g., potentille ansérine, Potentilla anserina $\mathrm{L}$.) étaient submergées. Par conséquent, l'habitat propice à la croissance de l'aster n'était que peu présent sur le site. Il est encore une fois difficile d'observer une tendance continue puisque seule l'étude de Houle et al. (2002) sert de point de comparaison pour cette population. Cependant, une diminution allant de plus de deux millions à seulement une centaine d'individus est jugée substantielle et même dramatique. Un autre relevé devrait être effectué dans les prochaines années afin de suivre l'état de cette population.

\section{Étang des caps}

Classe $6( \pm 240000$ individus $)$

Localisation : $\mathrm{N} 47^{\circ} 16.598^{\prime}$ à $\mathrm{N} 47^{\circ} 16.690^{\prime}$

\section{W61 ${ }^{\circ} 59.155^{\prime} \mathrm{W} 61^{\circ} 58.416^{\prime}$}

L'essentiel de cette population est situé sur un îlot dans la lagune du Havre aux Basques accessible à gué. Depuis l'étude de Houle et al. (2002), la population est caractérisée par un effectif très élevé. De toutes les populations visitées, celle-ci est présente l'effectif le plus important.

\section{Baie du portage \\ Classe 1 ( 8 individus) \\ Localisation : $\mathrm{N} 47^{\circ} 15.381^{\prime}$ \\ W61 $56.423^{\circ}$}

Cette population n'avait pas été revisitée depuis l'étude de Gagnon et al. (1995b) où l'effectif exact n'était pas indiqué. Le relevé de 2004 constitue donc la première évaluation de l'effectif de cette petite population.

Baie de Grosse-Île

Classe 5

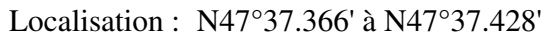

W61 $32.401^{\prime}$ à W61 $32.434^{\prime}$

La population est située le long d'un sentier de VTT. Le gradient de perturbation / compétition est donc entretenu par le passage récurrent de VTT. L'effectif de cette population a augmenté depuis la dernière observation par Houle et al. (2002). Il est possible que la popularité des VTT sur Grosse-Île (Alain Richard. Attention Frag' Îles, communication personnelle) encourage la croissance démographique de la population d'aster par le passage de nombreux appareils.

\section{Baie Clarke}

Classe 4 ( \pm 1000 individus)

Localisation : $\mathrm{N}^{\circ} 7^{\circ} 37.394^{\prime}$ à N47 $37.358^{\prime}$

W61 $28.331^{\prime} \mathrm{W} 61^{\circ} 28.368^{\prime}$

Cette population est également située le long d'un sentier de VTT et les sites favorables sont entretenus par leur passage récurrent. La population est stable depuis l'étude de Houle et al. (2002) et l'effectif a augmenté depuis les premières observations en 1995 (Gilbert et al. 1999).

\section{Pointe Old-Harry}

Classe 0. Bien que l'habitat semble propice, aucun individu n'a été retrouvé à ce site (station 6 de Houle et al. (2002)). Le suivi de Houle et al. (2002) notait une population stable de classe 2. Après une recherche exhaustive autour de la coordonnée géoréférencée fournie dans Houle et al. (2002), aucun individu n'a été retrouvé. Houle et al. (2002) notaient que l'ensemble de la végétation était fauchée pour entretenir un espace de stationnement, il est possible que la taille continuelle de cette plante annuelle a empêché l'établissement du stade adulte reproducteur menant ainsi cette petite population à l'extinction.

\section{Bassin aux Huîtres, bassin est}

Classe 3 ( \pm 500 individus)

Localisation : $\mathrm{N}^{\prime} 7^{\circ} 33.543^{\prime}$

W61 $30.304^{\prime}$

La population se trouve dans le gravier du stationnement. Houle et al. (2002) avait noté la présence de nombreuses tiges d'aster, constituant l'essentiel de la population près d'un étang. Aujourd'hui, l'étang s'est eutrophié et les algues vertes ont envahi les sites favorables à l'aster. La tendance à l'eutrophisation avait débuté lors du dernier relevé de Houle et al. (2002) (Gilles Houle, communication personnelle). Dans la présente étude, aucun aster n'a été retrouvé près de l'étang. Duclos (2004) avait concentré l'effort d'échantillonnage près de cet étang et n'avait pas noté la présence de l'aster dans le gravier (Geneviève Duclos, communication personnelle). La perte de cette partie de la population a entraîné un déclin, l'ensemble de la population passant de la classe 6 (Houle et al. 2002) à la classe 3 en 2004.

Bassin aux Huîtres, club vacance

Classe 2 ( \pm 50 individus)

Localisation : $\mathrm{N} 47^{\circ} 33.366^{\prime}$

W61 $30.936^{\prime}$

Les individus de cette population croissent à travers les galets d'une mise à l'eau pour embarcations nautiques. La population a conservé l'effectif noté par Houle et al. (2002).

\section{Bassin aux Huîtres, centre}

La population n'a pas été revisitée, une habitation privée avait été bâtie à proximité de la station de Houle et al. (2002). Cependant, lors du dernier relevé de Houle et al. (2002), il ne restait qu'un seul individu et Duclos (2004) n'en a noté aucun. Il est donc très possible que cette population soit maintenant éteinte.

\section{Bassin aux Huîtres, bassin ouest}

Classe 3 ( \pm 500 individus)

Localisation : $\mathrm{N}^{\circ} 7^{\circ} 32.613^{\prime}$ à N47 $32.656^{\prime}$

\section{W61 $31.848^{\prime} \mathrm{W} 61^{\circ} 31.946^{\prime}$}

Les individus sont répartis le long d'une bande à l'interface entre un milieu dunaire et une zone fréquemment inondée. La population semble en déclin continuel. Gilbert et al. (1999) et Houle et al. (2002) notaient une population de classe 6; dans Duclos (2004) elle passait vers la classe 4 et dans la présente étude une population de classe 3 est observée. Houle et al. (2002) ont noté une forte érosion de l'habitat de l'aster et une diminution importante en 2001 ne laissant qu'une population de classe 2. Il est donc possible que cette population soit soumise à de fortes perturbations cycliques et que l'effectif de cette population soit très variable.

Pointe de l'est

Cette population n'a pas été revisitée

\section{Élaboration d'un protocole de suivi simple}

Les trois populations retenues pour l'élaboration du protocole de suivi sont Barachois (BAR), Havre aux Basques (HAB) et Étang des caps (EDC). Les localisations exactes de chaque transect permanent ont été identifiées à l'aide d'un GPS et sont présentées au tableau 2. 
TABLEAU 2. Localisations des 15 transects permanents installés aux trois populations sélectionnées pour le suivi à long terme.

\begin{tabular}{|c|c|c|c|}
\hline Population & Transect & Latitude & Longitude \\
\hline \multirow[t]{5}{*}{ Barachois (BAR) } & BAR1 & $\mathrm{N} 47^{\circ} 25.369^{\prime}$ & $\mathrm{W} 61^{\circ} 51.682^{\prime}$ \\
\hline & BAR2 & $\mathrm{N} 47^{\circ} 25.400^{\prime}$ & $\mathrm{W} 61^{\circ} 51.703^{\prime}$ \\
\hline & BAR3 & $\mathrm{N} 47^{\circ} 25.432^{\prime}$ & W 61ํ⒌763' \\
\hline & BAR4 & $\mathrm{N} 47^{\circ} 25.448^{\prime}$ & W $61^{\circ} 51.962^{\prime}$ \\
\hline & BAR5 & $\mathrm{N} 47^{\circ} 25.442^{\prime}$ & $\mathrm{W} 61^{\circ} 52.012^{\prime}$ \\
\hline \multirow[t]{5}{*}{ Havre aux Basques (HAB) } & HAB 1 & $\mathrm{~N} 47^{\circ} 18.388^{\prime}$ & $\mathrm{W} 61^{\circ} 56.332^{\prime}$ \\
\hline & HAB2 & $\mathrm{N} 47^{\circ} 18.245^{\prime}$ & W $61^{\circ} 56.290^{\prime}$ \\
\hline & HAB3 & $\mathrm{N} 47^{\circ} 17.791^{\prime}$ & $\mathrm{W} 61^{\circ} 56.212^{\prime}$ \\
\hline & HAB 4 & $\mathrm{~N} 47^{\circ} 17.732^{\prime}$ & W $61^{\circ} 56.250^{\prime}$ \\
\hline & HAB5 & $\mathrm{N} 47^{\circ} 17.686^{\prime}$ & $\mathrm{W} 61^{\circ} 56.241^{\prime}$ \\
\hline \multirow[t]{5}{*}{ Étang des caps (EDC) } & EDC1 & $\mathrm{N} 47^{\circ} 16.707^{\prime}$ & W $61^{\circ} 58.905^{\prime}$ \\
\hline & EDC2 & $\mathrm{N} 47^{\circ} 16.719^{\prime}$ & W $61^{\circ} 58.877^{\prime}$ \\
\hline & EDC3 & $\mathrm{N} 47^{\circ} 16.717^{\prime}$ & W $61^{\circ} 58.871^{\prime}$ \\
\hline & EDC4 & $\mathrm{N} 47^{\circ} 16.702^{\prime}$ & W $61^{\circ} 58.764^{\prime}$ \\
\hline & EDC5 & $\mathrm{N} 47^{\circ} 16.687^{\prime}$ & $\mathrm{W} 61^{\circ} 58.626^{\prime}$ \\
\hline
\end{tabular}

Description de la situation actuelle aux populations sélectionnées pour le suivi

Des différences significatives entre les populations ont été notées pour toutes les variables à l'étude (Tableau 3). Aussi, pour quatre des six variables étudiées, il existe des différences entre les cinq transects d'une même population. L'absence de différence significative pour les deux autres variables serait attribuable au manque de degrés de liberté associé au modèle contenant de nombreuses données manquantes. En effet, il n'y avait aucune valeur de hauteur ou de nombre de capitules associés aux quadrats où il n'y avait pas d'aster. La figure 1A montre que la population Étang des caps (EDC) est la plus dense $(40.00 \pm 6.23$ tiges d'aster $\left./ 0.5 \mathrm{~m}^{2}\right)$ et est significativement supérieure à celle de Havre aux Basques (HAB) $(8.90 \pm 2.00$ tiges d'aster $/ 0.5 \mathrm{~m}^{2}$ ). La population Barachois (BAR) n'est pas différente des deux autres avec une valeur intermédiaire $\left(21.90 \pm 10.51\right.$ tiges d'aster $\left./ 0.5 \mathrm{~m}^{2}\right)$. La figure 1B montre que la population Étang des caps a un pourcentage de couverture par l'aster significativement supérieur $(6.00 \pm 0.98 \%)$ par rapport aux deux autres sites (HAB : $2.60 \pm 0.62 \%$ et BAR : $1.33 \pm 0.40 \%$ ). Cependant, à la figure 1C, on constate que la population Étang des caps a un pourcentage de couverture par l'ensemble de la végétation inférieur $(16.77 \pm 2.75 \%)$ par rapport aux deux autres populations (HAB : 52.57 $\pm 5.96 \%$ et BAR : $64.57 \pm 6.70 \%$ ). La hauteur des tiges d'aster ainsi que le nombre de capitules par individu présentent le même patron de variation entre les trois sites (Figures 1 D et E) : Havre aux Basques a les valeurs les plus élevées (respectivement $18.86 \pm 1.08 \mathrm{~cm}$ et $48.50 \pm 8.10$ capitules par plant). Étang des caps a des valeurs intermédiaires significativement différentes des deux autres populations (respectivement $11.63 \pm 0.74 \mathrm{~cm}$ et $25.93 \pm 3.17$ capitules par plant). Enfin, Barachois a les valeurs les plus basses (respectivement $8.41 \pm$ $0.84 \mathrm{~cm}$ et $6.12 \pm 1.41$ capitules par plant). Enfin, la figure $1 \mathrm{~F}$ montre que la richesse spécifique est plus élevée à Havre aux Basques $(7.17 \pm 0.59$ espèces /

TABLEAU 3. Résultats de l'ANOVA nichée pour évaluer les différences inter-populations et inter-transects pour les variables. Les valeurs $P$ en caractères gras sont significatives.

\begin{tabular}{|c|c|c|c|}
\hline IVariables & Facteurs & Valeurs $F$ & Valeurs $P$ \\
\hline \multirow[t]{2}{*}{ Nombre de tiges d'aster } & Population & 6.098 & 0.0035 \\
\hline & Transect (population) & 3.039 & 0.0016 \\
\hline \multirow[t]{2}{*}{ Pourcentage de couverture par l'aster } & Population & 15.069 & $<0.0001$ \\
\hline & Transect (population) & 3.227 & 0.0009 \\
\hline \multirow[t]{2}{*}{ Pourcentage de couverture par la végétation } & Population & 42.529 & $<0.0001$ \\
\hline & Transect (population) & 8.377 & $<0.0001$ \\
\hline \multirow[t]{2}{*}{ Hauteur des tiges d'aster } & Population & 24.925 & $<0.0001$ \\
\hline & Transect (population) & 1.831 & 0.066 \\
\hline \multirow[t]{2}{*}{ Nombre de capitules par aster } & Population & 9.612 & 0.0003 \\
\hline & Transect (population) & 0.878 & 0.574 \\
\hline \multirow[t]{2}{*}{ Richesse spécifique } & Population & 3.208 & 0.046 \\
\hline & Transect (population) & 7.441 & $<0.0001$ \\
\hline
\end{tabular}



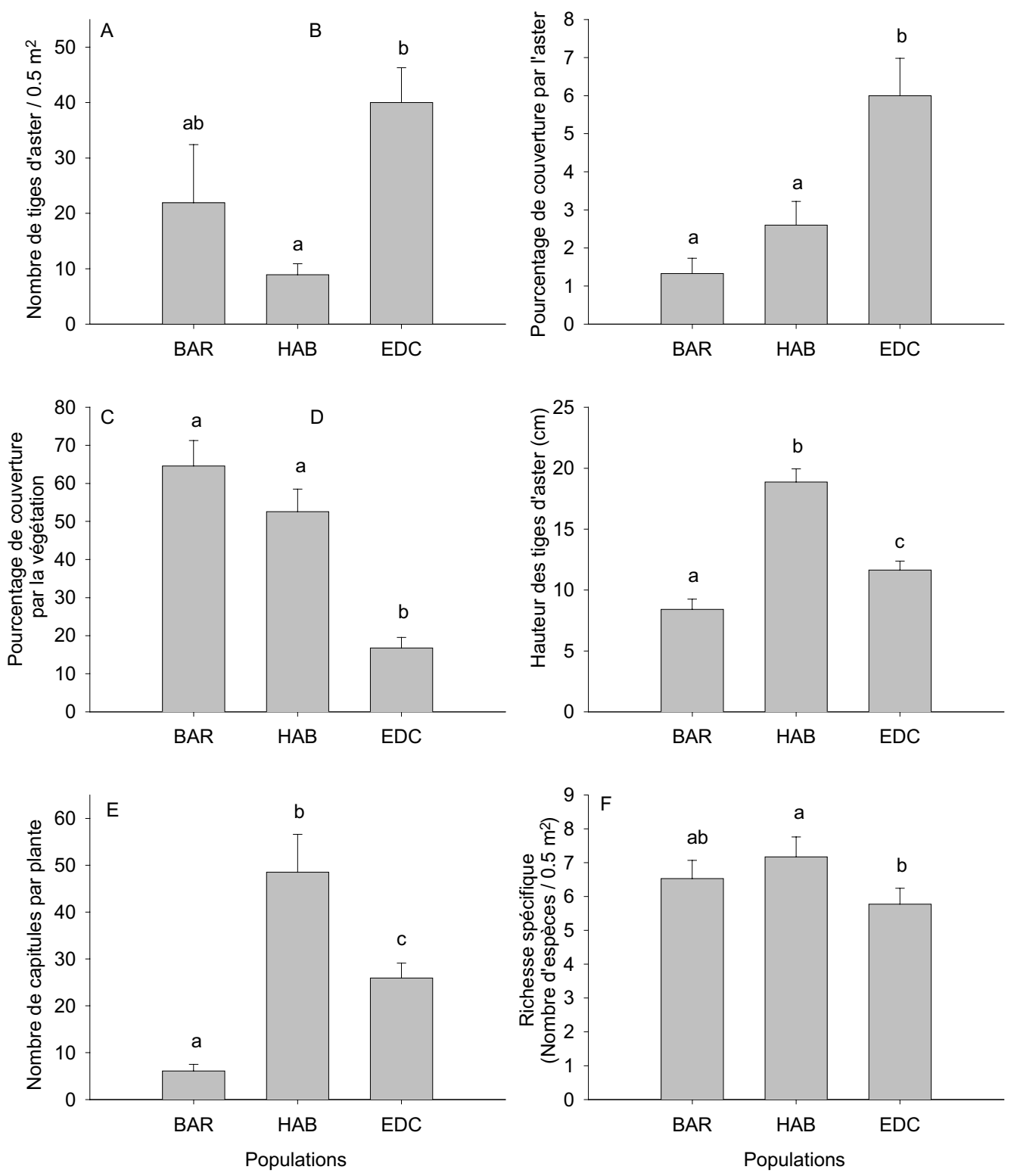

FIGURE 1. Variables à l'étude sur chacun des sites (moyenne par quadrat de $0.5 \mathrm{~m}^{2}+\mathrm{SE}$ ). Les lettres différentes au-dessus des colonnes représentent des valeurs moyennes significativement différentes $(P \leq 0.05)$ entre les sites.

$\left.0.5 \mathrm{~m}^{2}\right)$ qu'à Étang des caps $(5.77 \pm 0.47$ espèces / $0.5 \mathrm{~m}^{2}$ ), alors qu'elle est intermédiaire à la population Barachois $\left(6.53 \pm 0.54\right.$ espèces $\left./ 0.5 \mathrm{~m}^{2}\right)$.

Quoique l'ANOVA nichée démontre qu'il existe des différences significatives entre les transects à chacun des sites et que ces transects ne peuvent donc être considérés comme de véritables réplicats, on observe des relations significatives entre les variables et la distance par rapport à la ligne du rivage pour les populations HAB et EDC (Figure 2). La population
Barachois ne présente aucune tendance en fonction de la distance par rapport à la ligne du rivage. Les résultats complets sont présentés au tableau 4 et seules les régressions sélectionnées sont présentées dans le texte. Les figures $2 \mathrm{~A}$ et B montrent que le nombre d'aster et le pourcentage de couverture d'aster varient de façon quadratique (respectivement, HAB $\mathrm{R}^{2}=0.295, P=0.09$ et $E D C \mathrm{R}^{2}=0.218, P=0.036$; $\mathrm{HAB} \mathrm{R} \mathrm{R}^{2}=0.292, P=0.0095$ et $\mathrm{EDC} \mathrm{R}^{2}=0.280$, $P=0.012$ ) avec un mode aux distances intermédiaires 

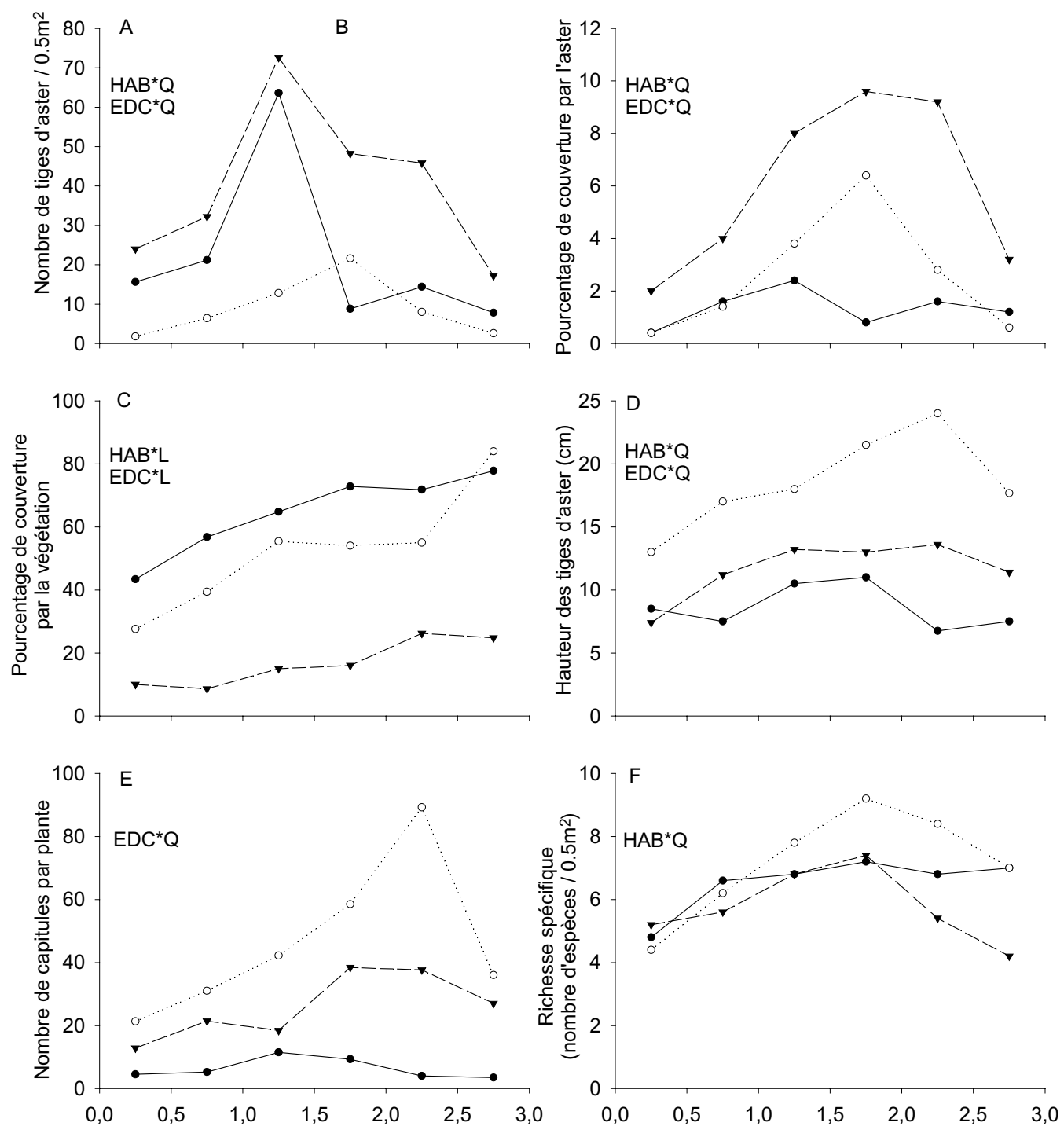

Distance par rapport à la ligne du rivage $(\mathrm{m})$

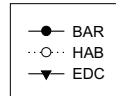

FIGURE 2. Variation des variables à l'étude le long du gradient topographique à chaque site. BAR* ${ }^{*}, \mathrm{HAB}^{*}$ ou EDC* indique une régression significative, suivie de L lorsque linéaire ou Q lorsque quadratique.

par rapport à la ligne du rivage (i.e. ces deux variables augmentent en s'éloignant du rivage pendant $1.5 \mathrm{~m}$ puis diminuent). Par contre, à la figure $2 \mathrm{c}, \mathrm{il}$ est démontré que le pourcentage de couverture de l'ensemble de la végétation augmente de façon linéaire et positive en s'éloignant de la ligne du rivage $\left(\mathrm{HAB} \mathrm{R} \mathrm{R}^{2}=0.247, P=0.0052\right.$ et $\mathrm{EDC} \mathrm{R}^{2}=0.177$, $P=0.021$ ). À la figure $2 \mathrm{~d}$, on voit que la hauteur des tiges d'aster varie de façon quadratique (HAB $\mathrm{R}^{2}=0.358, P=0.015$ et $\mathrm{EDC}^{2}=0.268, P=0.015$ ). La figure $2 \mathrm{e}$ montre que le nombre de capitules varie aussi de façon quadratique à la population Étang des caps $\left(\mathrm{R}^{2}=0.211, P=0.041\right)$, alors qu'à la figure $2 \mathrm{f}$ il est montré que la richesse varie de façon quadratique à Havre aux Basques $\left(\mathrm{R}^{2}=0.232, P=0.028\right)$.

\section{Discussion}

Mise à jour des données sur les effectifs, les localisations et les superficies des populations

L'étude de Gilbert et al. (1999) regroupe toutes les populations entourant la lagune du Havre aux Basques (Havre aux Basques, Pointe des véliplanchistes, Pointe 
TABLEAU 4. Régressions (linéaires : $y=\beta_{1} x+\beta_{0}$; et quadratiques $y=\beta_{2} x^{2}+\beta_{1} x+\beta_{0}$ ) des différentes variables $(y)$ en fonction de la distance par rapport à la ligne du rivage $(x)$. Les valeurs en caractères gras sont significatives $(P \leq 0.05)$ et $*$ indique que la régression a été sélectionnée lorsque les régressions linéaires et quadratiques étaient significatives.

\begin{tabular}{|c|c|c|c|c|}
\hline Variables & Populations & Régression & Coefficients de régression (RÇ) & Valeurs $P$ \\
\hline \multirow[t]{6}{*}{ Nombre de tiges d'aster } & \multirow[t]{2}{*}{ BAR } & Linéaire & 0.0097 & 0.605 \\
\hline & & Quadratique & 0.0366 & 0.605 \\
\hline & \multirow[t]{2}{*}{ HAB } & Linéaire & 0.0063 & 0.676 \\
\hline & & Quadratique & $0.295 *$ & $0.009 *$ \\
\hline & \multirow[t]{2}{*}{ EDC } & Linéaire & 0.0006 & 0.894 \\
\hline & & Quadratique & $0.218 *$ & $0.036 *$ \\
\hline \multirow[t]{6}{*}{ Pourcentage de couverture par l'aster } & \multirow[t]{2}{*}{ BAR } & Linéaire & 0.0029 & 0.777 \\
\hline & & Quadratique & 0.0300 & 0.663 \\
\hline & \multirow[t]{2}{*}{ HAB } & Linéaire & 0.0127 & 0.553 \\
\hline & & Quadratique & $0.292 *$ & $0.0095 *$ \\
\hline & \multirow[t]{2}{*}{ EDC } & Linéaire & 0.045 & 0.257 \\
\hline & & Quadratique & $0.280 *$ & $0.012 *$ \\
\hline \multirow[t]{6}{*}{ Pourcentage de couverture par la végétation } & \multirow[t]{2}{*}{ BAR } & Linéaire & 0.093 & 0.102 \\
\hline & & Quadratique & 0.101 & 0.238 \\
\hline & \multirow[t]{2}{*}{$\mathrm{HAB}$} & Linéaire & $0.247 *$ & $0.0052 *$ \\
\hline & & Quadratique & 0.249 & 0.021 \\
\hline & \multirow[t]{2}{*}{ EDC } & Linéaire & $0.177 *$ & $0.021 *$ \\
\hline & & Quadratique & 0.179 & 0.070 \\
\hline \multirow[t]{6}{*}{ Hauteur de l'aster } & \multirow[t]{2}{*}{ BAR } & Linéaire & 0.0069 & 0.751 \\
\hline & & Quadratique & 0.0066 & 0.619 \\
\hline & \multirow[t]{2}{*}{ HAB } & Linéaire & 0.221 & 0.027 \\
\hline & & Quadratique & $0.358 *$ & $0.015 *$ \\
\hline & \multirow[t]{2}{*}{ EDC } & Linéaire & 0.110 & 0.074 \\
\hline & & Quadratique & $0.268 *$ & $0.015 *$ \\
\hline \multirow[t]{6}{*}{ Nombre de capitules par plant } & \multirow[t]{2}{*}{ BAR } & Linéaire & 0.0071 & 0.748 \\
\hline & & Quadratique & 0.134 & 0.365 \\
\hline & \multirow[t]{2}{*}{ HAB } & Linéaire & 0.131 & 0.098 \\
\hline & & Quadratique & 0.194 & 0.129 \\
\hline & \multirow[t]{2}{*}{ EDC } & Linéaire & 0.159 & 0.029 \\
\hline & & Quadratique & $0.211 *$ & $0.041 *$ \\
\hline \multirow[t]{6}{*}{ Richesse spécifique } & \multirow[t]{2}{*}{ BAR } & Linéaire & 0.04 & 0.290 \\
\hline & & Quadratique & 0.065 & 0.404 \\
\hline & \multirow[t]{2}{*}{ HAB } & Linéaire & 0.104 & 0.083 \\
\hline & & Quadratique & $0.232 *$ & 0.028* \\
\hline & \multirow[t]{2}{*}{ EDC } & Linéaire & 0.0092 & 0.613 \\
\hline & & Quadratique & 0.142 & 0.126 \\
\hline
\end{tabular}

aux canots, Dune de l'ouest, Étang des caps et Baie du portage). Or, une seule station de Houle et al. (2002; station 19) montre un effectif dix fois supérieur à celui observé pour l'ensemble des populations entourant la lagune regroupées dans Gilbert et al. (1999). Il est possible que Houle et al. aient surestimé les populations par rapport aux autres études puisque dans l'étude de Houle et al. (2002), tous les relevés ont été effectués alors que les individus étaient au stade végétatif. Par conséquent, il est possible que Houle et al. aient inclus des plantules n'ayant pas encore émergé. Toutes les autres études se déroulant au mois de septembre, pendant la floraison, rapportent des effectifs un peu plus faibles que ceux de Houle et al. (2002). Cependant, il paraît peu probable que cette différence dans la phénologie explique à elle seule une diminution aussi importante que celle observée, par exemple, à la population de la dune de l'ouest (de plus de 2 millions à une centaine d'individus). Dans un souci d'uniformiser les futurs inventaires, les relevés subséquents devraient être effectués après la deuxième semaine d'août et avant la troisième semaine de septembre afin de tenir compte uniquement des individus adultes ayant émergé.

Les études de Gagnon et al. (1995a, 1995b) citées dans Gilbert et al. (1999) ont été réalisées au cours d'une année dont la période de croissance (mai à septembre) était particulièrement sèche (Archives Nationales d'information et de données climatologiques (Environnement Canada) données utilisées aux stations de Gaspé et Charlottetown (IPE) disponibles sur www. climate.weatheroffice.ec.gc.ca; tableau 5). Une étude sur les effets du stress hydrique sur différents stades de croissance de l'aster a démontré qu'une sécheresse avait un effet négatif sur l'établissement des plantules (Houle et Belleau 2000). Par conséquent, il est possible que les études de Gagnon et al. (1995a, 1995b) aient eu lieu 
TABlEaU 5. Tableau des données de pluviométrie mensuelle pour les saisons de croissance des années 1995 et 2004 et les moyennes mensuelles aux stations météorologiques de Gaspé et Charlottetown (IPE). Source : Archives Nationales d'information et de données climatologiques (Environnement Canada). ND indique que les données ne sont pas disponibles.

\begin{tabular}{|c|c|c|c|c|c|c|}
\hline \multirow[t]{2}{*}{ Site } & \multirow[t]{2}{*}{ Période } & \multicolumn{5}{|c|}{ Pluviométrie mensuelle (mm) } \\
\hline & & mai & juin & juillet & août & septembre \\
\hline \multirow[t]{3}{*}{ Gaspé } & 1995 & 45.0 & 32.9 & 82.0 & 71.2 & 28.6 \\
\hline & 2004 & 86.0 & 67.6 & 109.8 & 133.6 & 95.9 \\
\hline & Moyennes & 90.8 & 73.4 & 107.7 & 91.2 & 70.0 \\
\hline \multirow{3}{*}{$\begin{array}{l}\text { Charlottetown } \\
\text { (IPE) }\end{array}$} & 1995 & 697 & 962 & $59 ?$ & 593 & 625 \\
\hline & 2004 & ND & ND & ND & ND & $\mathrm{ND}$ \\
\hline & Moyennes & 97.7 & 93.2 & 85.8 & 87.3 & 95.4 \\
\hline
\end{tabular}

pendant une année où peu d'individus adultes avaient émergé entraînant des effectifs plus bas que la moyenne.

Au contraire, la présente étude s'est déroulée à la fin d'une saison de croissance où les précipitations ont été plus abondantes que la moyenne (Archives Nationales d'information et de données climatologiques (Environnement Canada) données utilisées à la station de Gaspé disponibles sur www.climate.weatheroffice.ec. gc.ca; tableau 5). Les niveaux d'eaux étant élevés, beaucoup de sites favorables à la croissance de l'aster étaient soit érodés (e.g., Pointe des véliplanchistes), soit perturbés par des dépôts de zostère (e.g., Pointe aux canots) ou soit complètement inondés (e.g., Dune de l'ouest). Ceci pourrait avoir eu pour effet de diminuer l'effectif des populations à des valeurs plus basses que la moyenne.

Les VTT semblent avoir un impact positif sur la croissance démographique de deux populations (Baie de Grosse-Île et Baie Clarke). Ceci peut être expliqué de deux façons. Premièrement, le passage récurrent des VTT peut créer le gradient de perturbation physique nécessaire à limiter la croissance des compétiteurs de l'aster du Saint-Laurent en permettant la croissance de celle-ci. La dynamique retrouvée le long des lagunes est conservée et les événements d'inondation sont remplacés par le passage d'un VTT. Deuxièmement, les VTT circulant sur de longues distances et créant un mouvement de l'air par leur passage peuvent faciliter la dissémination des akhènes d'aster qui pourra s'établir facilement sur le sol dénudé laissé par le passage des véhicules.

En résumé, les populations Cap de l'hôpital, Barachois, Havre aux Basques, Étang des caps, Baie de Grosse-Île, Baie Clarke et Bassin aux Huîtres (club vacance) sont stables ou en croissance. Les populations Barachois (Chemin Poirier), Pointe des véliplanchistes, Pointe aux canots, Dune de l'ouest et Bassin aux Huîtres (est et ouest) semblent décliner. Enfin, les populations Étang du Nord, Pointe Old-Harry, Bassin aux Huîtres (centre) semblent éteintes. Par contre, puisqu'il demeure difficile de départager entre une tendance continue au déclin et une dynamique variable selon les conditions annuelles, un suivi des populations à long terme demeure l'outil à privilégier.

\section{Élaboration d'un protocole de suivi simple}

Puisque plusieurs sites étaient fortement perturbés lors de la visite de 2004 et que plusieurs populations semblaient être caractérisées par des effectifs plus faibles que ceux observés dans d'autres études (e.g., populations de Dune de l'ouest ou Pointe des véliplanchistes), le suivi devra inclure une visite annuelle de toutes les populations. La revisite de l'ensemble des populations permettra de continuellement mettre à jour les données d'effectif, de localisation et de superficie des populations. Une revisite de ces populations pourra éventuellement permettre l'établissement de nouveaux transects permanents sur des populations importantes mais où il n'y avait pas de sites favorables en 2004. De plus, une attention particulière pourrait être portée sur les populations de Baie de Grosse-Île et de Baie Clarke qui subsistent et dont l'effectif semble même augmenter grâce à des perturbations d'origine anthropique liées au passage récurrent de VTT. Aucun transect permanent n'a été établi sur ces sites puisque les piquets auraient certainement nuit au passage des VTT et auraient été immédiatement retirés. Cependant, cette dynamique liée à la perturbation d'origine anthropique est intéressante et mérite que l'on s'y attarde à l'avenir.

Enfin, on peut s'attendre à ce que la ligne du rivage soit dépendante de la quantité de précipitations reçues. Par conséquent, il est fort possible que cette ligne varie et qu'il soit nécessaire de réajuster l'emplacement des transects au cours des prochaines années afin de couvrir adéquatement l'habitat de l'aster. Une façon de le faire est de visualiser chaque transect permanent comme deux lignes parallèles séparées par $1 \mathrm{~m}$ et d'une longueur indéfinie dont le premier quadrat (de $1 \mathrm{~m} \times 0.5 \mathrm{~m}$ ) est à proximité de la ligne du rivage et le dernier est situé $2.5 \mathrm{~m}$ plus loin pour donner un transect d'échantillonnage d'une longueur totale constante de $3 \mathrm{~m}$. Si, au cours des prochaines années, il est noté que la ligne du rivage se déplace, les transects installés actuellement serviront de bornes indiquant les deux lignes parallèles; il ne suffira que 
de tirer des lignes continues jusqu'à la ligne du rivage pour y installer le premier quadrat. Si une telle démarche doit être utilisée, il faudrait inclure dans le suivi la mesure de la distance de déplacement du transect par rapport au transect permanent original.

Description de la situation actuelle aux populations sélectionnées pour le suivi

Les résultats ont montré que les trois populations choisies pour le suivi à long terme présentent des différences significatives pour toutes les variables incluses dans le suivi. La population Étang des caps présente la plus forte densité et les individus de cette population couvrent un plus grand pourcentage de sol. Ces résultats soutiennent les estimés de l'effectif où Étang des caps était considérée comme la seule population de classe 6 . Ceci peut être dû à une faible compétition interspécifique, puisque les résultats de l'analyse ont montré qu'il s'agit du site où le pourcentage de l'ensemble de la végétation et la richesse spécifique sont les plus faibles. Ceci indique donc une différence au niveau de la dynamique de cette population par rapport aux deux autres populations. Cette dynamique favorise la croissance de l'aster par rapport aux autres espèces.

Les individus de la population Havre aux Basques ont atteint en 2004 une taille plus haute et possèdent davantage de capitules alors que les individus de la population Barachois étaient, au contraire, les plus petits et les moins ramifiés. La densité de la population du Barachois est deux fois plus élevée que celle observée au Havre aux Basques. Il est intéressant de constater que toutes les autres variables mesurées à ces deux sites ne présentent pas de différences significatives. Ces deux populations semblent donc utiliser deux stratégies morphologiques distinctes. La population du Barachois double la quantité de tiges mais réduit la taille et le nombre de capitules de celles-ci, alors que la population du Havre aux Basques, moins dense est composée d'individus plus hauts et ramifiés. Ces deux stratégies permettent d'obtenir le même pourcentage de couverture par l'aster. Il semble que la compétition interspécifique ne soit pas reliée à l'utilisation de l'une ou l'autre de ces stratégies puisque les deux populations sont situées sur des sites ayant une même richesse spécifique et un même pourcentage de couverture de végétation. L'utilisation de stratégies distinctes doit donc être reliée à une variable qui n'a pas été mesurée dans le suivi telle que la salinité du substrat qui inhibe la germination (Houle et al. 2001) ou une période de sécheresse en début de saison de croissance qui retarde l'établissement des plantules (Houle et Belleau 2000).

Il appert que les trois populations possèdent des différences qui renseignent sur l'importance de la densité, la morphologie et l'habitat. Par conséquent, il est fortement suggéré de conserver ces trois populations et ces six variables dans le suivi à long terme afin de vérifier dans quelle mesure ces populations conservent les mêmes caractéristiques au cours des années subséquentes ou si la dynamique est sujette à changer (e.g., en fonction de la quantité de précipitations). Aussi, tel que mentionné plus haut, l'inclusion de nouvelles populations est recommandée, particulièrement celles dont les effectifs varient entre les études (e.g., Dune de l'ouest, Pointe des véliplanchistes, Pointe aux canots).

Pour quatre des six variables, il existe des différences significatives entre les cinq transects de chaque population. Ceci suggère que les transects ne sont pas des réplicats et qu'ils doivent plutôt être considérés comme différents micro-habitats. Par conséquent, il est important de conserver les quinze transects dans le suivi à long terme. Retirer un transect constituerait une perte significative d'information. Les données recueillies au cours des prochaines années du suivi, permettront en plus de comparer la progression d'un même transect au cours du temps. Ceci permettra de voir comment chaque micro-habitat se transforme.

Même si les transects ne sont pas de véritables réplicats, il est possible de discerner des relations significatives des différentes variables en fonction de la distance par rapport à la ligne du rivage pour deux populations. Le plan d'échantillonnage de transects organisés perpendiculairement à la ligne de rivage sur une distance de $3 \mathrm{~m}$ permet de couvrir le gradient de perturbation / compétition attendu sur deux sites. En effet, aux populations de HAB et EDC, le nombre de tiges et le pourcentage de couverture par l'aster de même que les valeurs associées aux variables morphologiques (la hauteur des tiges et le nombre de capitules par individu) sont faibles près de la ligne du rivage où le stress lié à la salinité (Houle et al. 2001; Reynolds et al. 2001) et les perturbations (Reynolds et Houle 2002) empêchent la croissance de l'aster. En s'éloignant du rivage, les conditions du milieu favorisent la croissance de l'aster mais demeurent trop restrictives pour la croissance d'espèces compétitrices. Le mode de la distribution se situe dans cette bande intermédiaire. À une distance plus éloignée de la rive, les conditions deviennent favorables pour des espèces plus compétitrices qui limitent la croissance de l'aster (Brumbt 2001; Houle et Valéry 2003). Ce gradient est confirmé par le pourcentage de couverture par l'ensemble de la végétation qui augmente de façon linéaire à partir de la ligne du rivage. La richesse spécifique suit la distribution quadratique à la population Havre aux Basques. Il semble que cette relation soit un artéfact de la relation entre diversité et productivité fréquemment observée (Grime 1973; Rosenzweig 1992; Huston et deAngelis 1994; de Lafontaine 2004). En effet, le maximum de richesse est observé à la distance correspondant à des valeurs intermédiaires de pourcentage de couverture de l'ensemble de la végétation.

Par contre, à la population Barachois, le plan d'échantillonnage ne permet pas de déceler le gradient de perturbation / compétition attendu. Le site est particulier et les transects T4 et T5 ont été positionnés un 
peu en retrait par rapport à la ligne du rivage puisque la plupart des individus ne sont pas situés dans l'habitat typique de la bande de $3 \mathrm{~m}$ après la ligne de rivage. Il est possible de redéfinir l'emplacement de ces transects afin qu'ils correspondent plus adéquatement au gradient typique. Il se peut que cette démarche uniformise les transects à ce site créant ainsi de véritables réplicats. Cependant, tant que ces transects seront peuplés d'aster, il est plutôt conseillé de les conserver tels qu'ils sont présentement en tenant compte de cette particularité dans le suivi à long terme.

\section{Conclusions}

Cette étude définit 18 populations. Les 7 populations Cap de l'hôpital, Barachois, Havre aux Basques, Étang des caps, Baie de Grosse-Île, Baie Clarke et Bassin aux Huitres (club vacance) sont stables ou en croissance. Les 6 populations Barachois (Chemin Poirier), Pointe des véliplanchistes, Pointe aux canots, Dune de l'ouest et Bassin aux Huîtres (est et ouest) semblent décliner. Enfin, les 3 populations Étang du Nord, Pointe Old-Harry, Bassin aux Huîtres (centre) semblent éteintes ou très près de l'extinction. La population Pointe de l'est n'a pas été revisitée et cette étude présente le premier estimé de la population Baie du portage. À partir des études qui ont été réalisées en utilisant différentes approches, il est difficile de départager entre de réelles tendances au déclin et une dynamique en fonction des conditions changeantes du milieu. L'utilisation d'un suivi à long terme appliquant un protocole uniforme permettra de déterminer la progression des différentes populations.

Un protocole a été élaboré et le plan d'échantillonnage a été installé à trois populations : Barachois, Havre aux Basques et Étang des caps. Il est conseillé d'installer le plan d'échantillonnage sur d'autres sites importants où les effectifs semblent en déclin mais sur lesquels il était impossible d'établir des transects en 2004 (Dune de l'ouest, Pointe aux canots, Pointe des véliplanchistes). Il serait également intéressant d'étudier la dynamique des populations d'aster perturbées par le passage récurrent des VTT (Baie de Grosse-île et Baie Clarke). Enfin, il est recommandé d'effectuer une visite annuelle des 18 populations.

$\mathrm{Au}$ sein des trois populations sélectionnées pour le suivi, les résultats obtenus en 2004 montrent des différences significatives pour toutes les variables considérées. Aux trois sites, les transects présentent des différences entre eux et ne peuvent donc pas être considérés comme des réplicats. Â la lumière de ces informations, il est recommandé de conserver les trois populations pour le suivi à long terme et de conserver les cinq transects tels qu'ils se présentent actuellement. Le plan d'échantillonnage permet de déceler le gradient de perturbation / compétition attendu sauf à la population plus particulière du Barachois. Ceci indique que pour les populations de Havre aux Basques et Étang des caps, ce plan d'échantillonnage permet de tenir compte de la variation par rapport à la ligne du rivage et devrait être conservé comme tel. Cependant, les transects 4 et 5 de la population du Barachois ont été déplacés à cause de contraintes particulières associées à ce site. Ces transects devraient être laissés ainsi et l'analyse devra en tenir compte.

\section{Remerciements}

Le soutien financier a été fourni par le ministère du développement durable, environment et parcs du Québec et Environnement Canada. Les données ont été fournies par le ministère du développement durable, environment et parcs du Québec .

\section{Littérature citée}

Brumbt, C. P. 2001. Effet de la compétition interspécifique sur l'aster du Saint-Laurent (Aster laurentianus Fernald) aux Îles-de-la-Madeleine, Québec. Mémoire de maîtrise. Université Laval, Québec. 56 pages.

COSEPAC. 2004. Espèces canadiennes en péril, mai 2004. Comité sur la situation des espèces en péril au Canada. 57 pages.

de Lafontaine, G. 2004. Richesse spécifique le long d'un gradient de production: utilisation d'une approche multivariée. Mémoire de maîtrise. Université Laval, Québec. 44 pages.

Duclos, G. 2004. Suivi des populations de Symphyotrichum laurentianum, une plante endémique du sud du Golfe du Saint-Laurent. Thèse d'initiation à la recherche. Université de Moncton, Moncton. 35 pages.

Gagnon, J., G. Lavoie, G. Jolicoeur, et F. Boudreau. 1995a. Les plantes susceptibles d'être désignées menacées ou vulnérables de l'île de l'Est, Îles-de-la-Madeleine. Gouvernement du Québec, ministère de l'Environnement et de la Faune, Direction de la conservation et du patrimoine écologique, Québec. 33 pages.

Gagnon, J., G. Lavoie, G. Jolicoeur, et F. Boudreau. 1995b. Les plantes susceptibles d'être désignées menacées ou vulnérables de la lagune du Havre aux Basques, Îles-de-laMadeleine. Gouvernement du Québec, ministère de l'Environnement et de la Faune, Direction de la conservation et du patrimoine écologique, Québec. 25 pages.

Gilbert, H., J. Labrecque, et J. Gagnon. 1999. La situation de l'aster du Saint-Laurent (Aster laurentianus, syn. : Symphyotrichum laurentianum) au Canada. Gouvernement du Québec, ministère de l'Environnement, Direction de la conservation et du patrimoine écologique, Québec. 34 pages.

Grime, J. P. 1973. Competitive exclusion in herbaceous vegetation. Nature 242: 344-347.

Grime, J. P. 1977. Evidence for the existence of three primary strategies in plants and its relevance to ecological and evolutionary theory. American Naturalist 111: 11691194.

Grime, J. P. 1979. Plant strategies and vegetation processes. Wiley, Chichester, United Kingdom.

Guignion, M., C. Ristau, et D. Lemon. 1995. The distribution and abundance of the Gulf of St. Lawrence Aster, Aster laurentianus, in Prince Edward Island National Park. Canadian Field-Naturalist 109: 462-464.

Houle, F., et E. Haber. 1990. Status of the Gulf of St. Lawrence Aster, Aster laurentianus (Asteraceae), in Canada. Canadian Field-Naturalist 104: 455-459.

Houle, G., et A. Belleau. 2000. The effects of drought and waterlogging conditions on the performance of an endemic 
annual plant, Aster laurentianus. Canadian Journal of Botany 78: 40-46.

Houle, G., C. P. Brumbt, et C. E. Reynolds. 2002. Écologie de l'aster du saint-laurent, Aster laurentianus, aux Îlesde-la-Madeleine. Gouvernement du Québec, ministère de l'Environnement, Direction de la conservation et du patrimoine écologique, Québec. 162 pages.

Houle, G., L. Morel, C. E. Reynolds, et J. Siégel. 2001. The effect of salinity on different developmental stages of an endemic annual plant, Aster laurentianus (Asteraceae). American Journal of Botany 88: 62-67.

Houle, G., et S. Valéry. 2003. A mixed strategy in the annual endemic Aster laurentianus (Asteraceae) - a stress tolerant, yet opportunistic species. American Journal of Botany 90: 278-283.

Huston, M. A., et D. L. deAngelis. 1994. Competition and coexistence: the effects of resource transport and supply rates. American Naturalist 144: 954-977.
Reynolds, C. E., et G. Houle. 2002. Mantel and partial Mantel tests suggest some factors that may control the local distribution of Aster laurentianus at Îles de la Madeleine, Québec. Plant Ecology 164: 19-27.

Reynolds, C. E., G. Houle, et C. Marquis. 2001. Light and salinity affect growth of the salt marsh plant Aster laurentianus. New Phytologist 149: 441-448.

Rosenzweig, M. L. 1992. Species diversity gradients: we know more and less than we thought. Journal of Mammalogy 73: 715-730.

Sokal, R. R., et F. J. Rohlf. 1969. Biometry. W. H. Freeman and Company, San Francisco.

Received 24 January 2005

Accepted 13 October 2005 